\title{
Same Origin Three-Dimensional Strain Detection FBG Sensor Based on Elliptical Ring and Its Optimization
}

\author{
Shanchao JIANG, Jing WANG*, Qingmei SUI, and Qinglin YE \\ School of Control Science and Engineering, Shandong University, Jinan, 250061, China \\ *Corresponding author: Jing WANG \\ E-mail: wangjingkz@sdu.edu.cn
}

\begin{abstract}
In order to achieve the same origin three-dimensional (3D) strain measurement, one three-dimensional (3D) fiber Bragg grating (FBG) strain sensor is proposed in this paper. The metal structure of this sensor is composed by three elliptical rings with different geometrical parameters. All these elliptical rings make sure that this sensor achieves the same origin 3D strain detection and increases the strain measurement coefficient. A theory calculation model of this sensor is established. The finite element method is utilized to optimize this sensor and verify the correctness of the theory model. After sensor optimization, $1 \mathrm{~mm}$ is chosen as the radical thickness of this sensor based on taking high strain detection coefficient and structure strength into account. To further obtain detection characteristics of this sensor, the calibration experiment is carried out. Experimental data of FBG1 which is the core sensitive element of this sensor is chosen as the specimen to be analyzed by the least square method. When the wavelength of FBG1 is changed by external stress, wavelengths of FBG2 and FBG3 have just a little fluctuation maybe caused by the fiber demodulation instrument SM125. So sensitive elements (FBG1, FBG2, and FBG3) of this sensor have no crosstalk problem for three-dimensional detection. After data analysis, the measuring coefficient of FBG1 is $0.05 \mathrm{~nm} / \mathrm{N}$. Similarly, the coefficients of FBG2 and FBG3 are $0.045 \mathrm{~nm} / \mathrm{N}$ and $0.39 \mathrm{~nm} / \mathrm{N}$, respectively. All these data confirm that this sensor could achieve the same origin $3 \mathrm{D}$ strain measurement without the crosstalk problem and has certain practical applications.
\end{abstract}

Keywords: Fiber Bragg grating, 3D strain measurement, finite element method, sensor optimization

Citation: Shanchao JIANG, Jing WANG, Qingmei SUI, and Qinglin YE, "Same Origin Three-Dimensional Strain Detection FBG Sensor Based on Elliptical Ring and Its Optimization," Photonic Sensors, 2015, 5(2): 146-151.

\section{Introduction}

Strain at the particular point of the massive structure, such as the dam, soil basement, and tunnel, varies in all directions. All these strains contain the significant information about the engineering structure safety $[1,2]$. Therefore, interior strain information is needed [3] to investigate the health condition of the civil structure. However, conventional strain monitoring methods, such as strain gauge, can not measure multi-dimensional interior strain of the engineering structure effectively. Due to its unique advantages including immunity to electromagnetic interference, compact size, resistance to corrosion, high sensitivity, and the ability to be embedded into composites with minimal structural degradation, the optical fiber strain sensor is widely used in many applications [4-7]. In principle, with adequate sampling and signal processing, the optical fiber strain sensor is

Received: 16 January 2015 / Revised version: 16 March 2015

(C) The Author(s) 2015. This article is published with open access at Springerlink.com

DOI: $10.1007 / \mathrm{s} 13320-015-0240-5$

Article type: Regular 
usually more accurate than electrical resistive strain gauge. So the optical fiber strain sensor provides one promising measurement for multi-dimensional strain detection. To date, the optical fiber strain sensors can be divided into integral and point sensors. Most of them still can only give the strain distribution in planar condition [8]. Through the existed embedding methods such as three-dimensional (3D) coordinate and tetrahedron methods, many fiber strain sensors could realize 3D strain measurement. But achieving 3D strain measuring through three independent strain sensors has many disadvantages such as vertical controlling of three axes, difference detection origin, and signal integrity. In order to solve all these disadvantages, one fiber Bragg grating (FBG) strain sensor which achieves the same origin 3D strain measurement simultaneously is urgently needed.

This paper proposes one FBG based sensor assembly which could achieve the same origin $3 \mathrm{D}$ strain measurement. A theory calculation model is built up based on the mechanical basic theory. The metal structure of this 3D FBG strain sensor includes three elliptical rings with different geometrical parameters. All these different parameters make sure that this sensor can achieve the same origin $3 \mathrm{D}$ strain measurement. Usually, the strain sensitivity of the bare FBG is about $1 \mathrm{pm} / \mu \varepsilon$ which is too small to be used in practical monitoring [9]. All these three elliptical rings can also achieve sensitization function of strain detection. In order to take its measurement sensitivity and structure strength into account, the finite element method is utilized to optimize this sensor, and the correctness of the theory calculation model is also verified in this stage. To further obtain characteristics of this sensor, its calibration experiment under different stresses has been operated. The experimental result shows that the proposed 3D strain sensor assembly can achieve the same origin 3D strain measurement accurately with out crosstalk problems.

\section{Sensor structure and sensing principle}

\subsection{Sensor structure}

The 3D FBG strain sensor which is proposed in this paper is composed by one metal structure and three FBGs which are core sensitive elements. The design diagram of this sensor is shown in Fig. 1. The metal structure is made up by three elliptical rings with different geometrical parameters. All these parameters make sure that three FBGs included in this sensor have the same detection origin. A crossbeam in the elliptical ring acts as a substrate and achieves strain transitive. Due to the function of crossbeams, the space position of each ring is fixed. The basic geometry parameters of these three elliptical rings are displayed in Table 1.

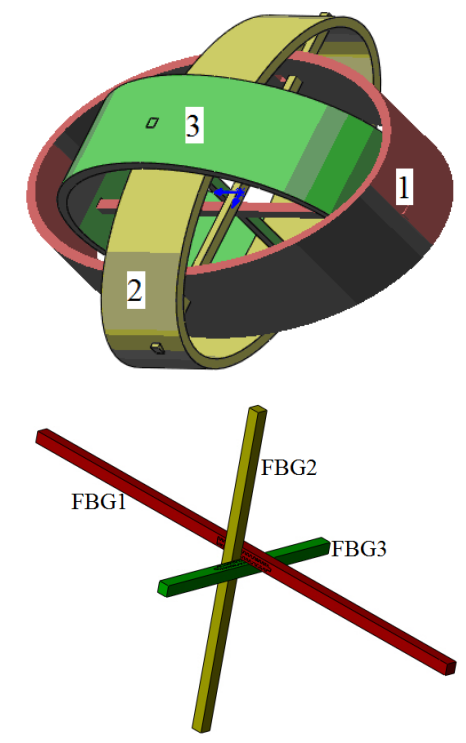

Fig. $13 \mathrm{D}$ assembly drawing of this FBG strain sensor.

Table 1 Basic geometry parameters of three elliptical rings (unit: $\mathrm{mm}$ ).

\begin{tabular}{ccccc}
\hline $\begin{array}{c}\text { No. of } \\
\text { elliptical ring }\end{array}$ & Major axis & Minor axis & Height & Radical thickness \\
\hline 1 & 46 & 26 & 10 & 1 \\
2 & 38 & 24 & 10 & 1 \\
3 & 38 & 18 & 10 & 1 \\
\hline
\end{tabular}

\subsection{Sensing principle}

The FBG has wavelength selection characteristics, and only the wavelength that 
satisfies the Bragg condition is affected and strongly back-reflected. The reflected central wavelength $\lambda_{B}$ could be expressed as [10]

$$
\lambda_{B}=2 n_{\text {eff }} \Lambda
$$

where $n_{\text {eff }}$ is the effective index of refraction, and $\Lambda$ is the grating periodicity of the FBG.

Due to the influence of temperature and strain on parameters $n_{\text {eff }}$ and $\Lambda$, the FBG reflected wavelength is changed as the function of temperature and strain. External temperature is kept stable, and the general relationship between the strain $\varepsilon$ and wavelength shift $\Delta \lambda_{B}$ can be described as [11]

$$
\frac{\Delta \lambda_{\varepsilon}}{\lambda_{B}}=\left(1-p_{e}\right) \varepsilon
$$

in which $p_{e}$ is the optical elasticity coefficient of the fiber.

The theory calculation model of this sensor is built up according to mechanical basic knowledge. As the elastic modulus of the crossbeam is much smaller than that of the elliptical ring, the function of the crossbeam is ignored in the theory calculation model. The elliptical ring 1 is chosen as a specimen to calculate strain changes under different stresses of this 3D FBG strain sensor theoretically. Its overhead view is exhibited in Fig. 2.

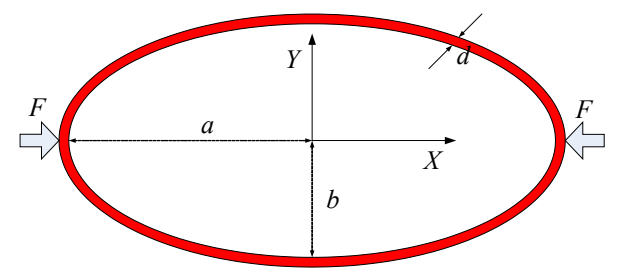

Fig. 2 Overhead view of the elliptical ring 1.

As the external stress is $F$, surface strain $\varepsilon$ of the crossbeam included in the elliptical ring 1 could be expressed as

$$
\varepsilon=\frac{\pi^{2}-8}{8 \pi a} \frac{F b^{3}}{E I}
$$

where $E$ is the elastic modulus, $I$ is the geometrical moment of inertia, and $a$ and $b$ are half the length of the major and minor axes of the elliptical ring 1, respectively.

$$
I=\frac{1}{12} h d^{3}
$$

in which $h$ and $d$ represent the height and radical thickness of the ring.

So the relationship between the wavelength shift and stress is given in (5).

$$
\Delta \lambda_{B}=\lambda_{B} \cdot \frac{\pi^{2}-8}{8 \pi a} \frac{b^{3}}{E I}\left(1-p_{e}\right) \cdot F .
$$

Hence, once the geometry and material parameters of this sensor have certain values, the correspondence obtained from the theory calculation model of this sensor shows the perfect linearity.

\section{Sensor optimization}

Different radial thicknesses should be considered to make sure that this sensor has high strain measuring precision with certain mechanical strength. So, the finite element method (FEM) [12] is introduced to optimize and identify the relationship between the radial thickness and structure strain of this 3D sensor. The elliptical ring 1 is chosen as a specimen to achieve this sensor optimization. A 3D solid of the elliptical ring 1 is built up based on software Solidworks 2012 [13]. The major and minor axes of the elliptical ring 1 are $46 \mathrm{~mm}$ and $26 \mathrm{~mm}$, and its elastic modulus is $210 \mathrm{GPa}$. The longitudinal stria wood is chosen as a material of the crossbeam, and its elastic modulus is $10 \mathrm{GPa}$. Figure 3 gives that the surface strain distribution of the elliptical ring 1 refers to the radical thickness $1 \mathrm{~mm}$ and external stresses $20 \mathrm{~N}$ and $40 \mathrm{~N}$. The large surface strain almost distributes around the stress working area, and the strain in the other area has the same values. The concentrated distribution of the surface strain can ensure the coefficient of strain transitive. So the metal structure of this 3D FBG sensor can achieve optical sensibilization effect with a high strain transitive coefficient.

The external stress is fixed at $30 \mathrm{~N}$ in this optimization as considering the theory calculation of this sensor and maximum wavelength shift of FBG 
$8 \mathrm{~nm}$. Figure 4 shows the correspondence relationship between the longer axis strain of the elliptical ring 1 and its radial thickness.

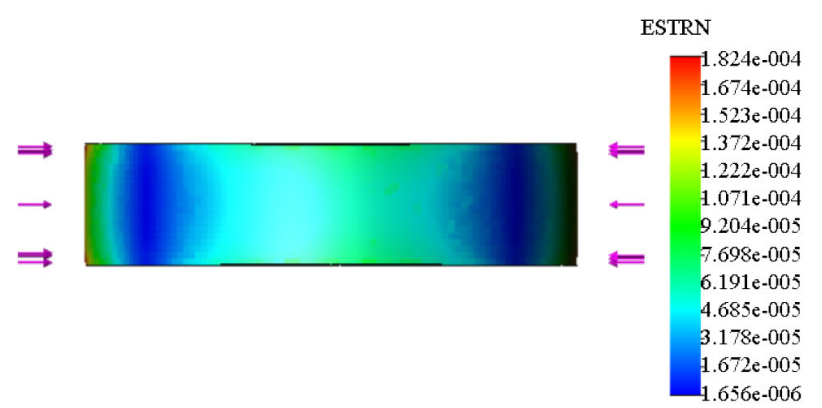

(a)

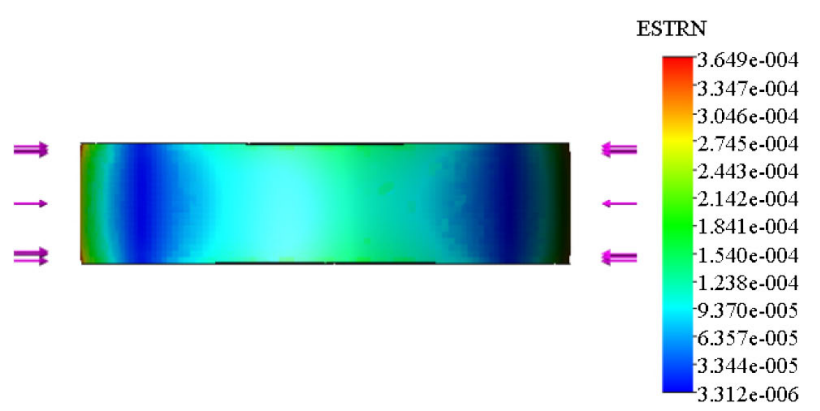

(b)

Fig. 3 Surface strain distribution of the elliptical ring 1 under different stresses: (a) $20 \mathrm{~N}$ and (b) $40 \mathrm{~N}$.

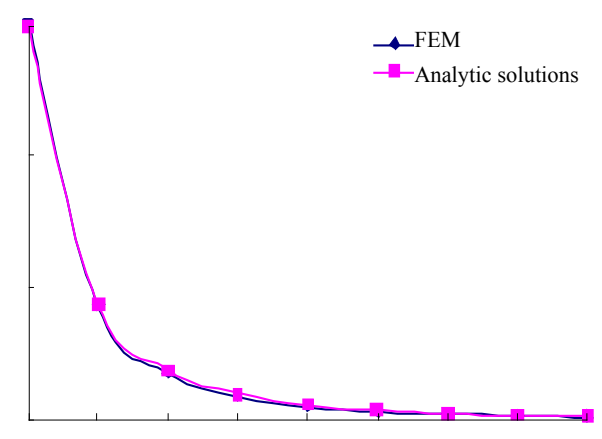

Fig. 4 Correspondence relationship between the major axis strain and radical thinness.

Taking detection accuracy and enough structure strength of this $3 \mathrm{D}$ strain sensor into account, $1 \mathrm{~mm}$ is chosen as the prototype radical thickness of this sensor.

\section{Calibration experiment}

To obtain the characteristics of this 3D strain sensor, its calibration experiment is carried out in this section.

\subsection{Experimental platform}

The calibration experimental platform shown in Fig. 5 is built up based on the sensor prototype, SM125 [14] (demodulation wavelength range: $1510 \mathrm{~nm}$ - $1590 \mathrm{~nm}$, precision: $1 \mathrm{pm}$ ) and load measuring instrument (precision $0.05 \mathrm{~N}$ ). Initial central wavelengths of the FBG in this $3 \mathrm{D}$ strain sensor are $1540.0858 \mathrm{~nm}$ (the elliptical ring 1, FBG1), $1549.9904 \mathrm{~nm}$ (the elliptical ring 2, FBG2), and $1539.9085 \mathrm{~nm}$ (the elliptical ring 3, FBG3).

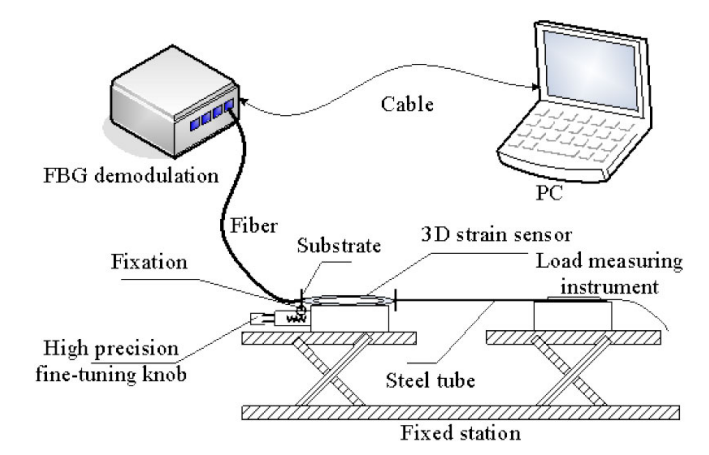

Fig. 5 Block diagram of the calibration experimental platform.

\subsection{Uniaxial strain experiment and data analysis}

At the beginning of this experiment, the elliptical ring 1 is fixed on the station, and the other two rings are kept at a free-form state. The high precision fine-tuning knob is adjusted to manually control external load changes with an interval step of $-0.05 \mathrm{~N}$. The range of the external load is from $-1 \mathrm{~N}$ to $0 \mathrm{~N}$. The force direction is chosen as negative when the sensor is compressed. Then, the stress state of the other two rings is changed in turn under the same experimental process. Data of FBG1 (the elliptical ring 1) are chosen as the specimen in data analysis. Figure 6 shows the relationship between the external loading and wavelength shift.

The central wavelengths of FBG2 and FBG3 fluctuate vary small which maybe are caused by fiber demodulation instrument SM125. When the external loadings are bigger than $-0.4 \mathrm{~N}$, wavelength shifts of the elliptical ring 1 in this experiment are almost the same with the theory calculation values. 
When the exterior loadings are less than $-0.4 \mathrm{~N}$, the relative errors between the experiment and theory are all less than 5\%. After data analysis by the least square method, the strain measurement coefficient of the elliptical ring 1 is $0.05 \mathrm{~nm} / \mathrm{N}$. The relative error of this coefficient between the theory calculation and experiment is $2.51 \%$. All these relative errors are caused by the thoughtless function of the crossbeam elastic modulus in theory calculation and micro-bend of the crossbeam under the external loading. Similarly, strain measurement coefficients of the elliptical rings 2 and 3 are $0.045 \mathrm{~nm} / \mathrm{N}$ and $0.39 \mathrm{~nm} / \mathrm{N}$, respectively. All these data verify that this $3 \mathrm{D}$ sensor can achieve $3 \mathrm{D}$ strain measurement without the crosstalk problem.

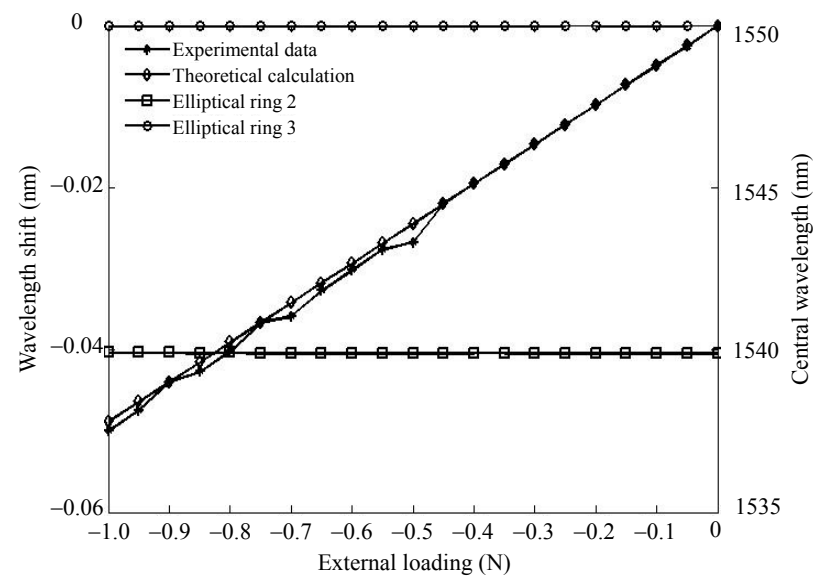

Fig. 6 Wavelength shift of FBG1 follows the external loading with the central wavelengths of FBG2 and FBG3.

\section{Conclusions}

One novel 3D FBG strain sensor is proposed in this paper, which can achieve the same origin 3D strain measurement based on three core sensitive elements (FBG1, FBG2, and FBG3). Based on the function of its metal structure which is composed by three different geometrical parameter elliptical rings, 3D strain detected by this sensor has the same measurement origin. The design of this sensor solves the problem that multi-dimensional interior strain measurement usually has different detection origins. The calculation model of this sensor is established and further verified in sensor optimization by the FEM. Taking the strain measurement coefficient and structure strength into account after sensor optimization, $1 \mathrm{~mm}$ is chosen as the radical thickness of this sensor prototype. The calibration experiment is carried out to check the characteristics of this sensor prototype. Through data analysis, wavelengths of FBG2 and FBG3 are not affected when FBG1 is influenced by the external stress. So sensitive elements of this sensor have no crosstalk problem. Using the least square method to deal with experiment data, the strain measurement coefficients of this sensor are $0.05 \mathrm{~nm} / \mathrm{N}$ (FBG1), $0.045 \mathrm{~nm} / \mathrm{N}$ (FBG2), and $0.39 \mathrm{~nm} / \mathrm{N}$ (FBG3). All these data confirm that this sensor can achieve the same origin 3D strain measurement with high detection coefficients and has certain practical applications.

Open Access This article is distributed under the terms of the Creative Commons Attribution License which permits any use, distribution, and reproduction in any medium, provided the original author(s) and source are credited.

\section{References}

[1] M. Perry, Z. Yan, Z. Sun, L. Zhang, P. Niewczas, and M. Johnston, "High stress monitoring of prestressing tendons in nuclear concrete vessels using fibre-optic sensors," Nuclear Engineering and Design, 2014, 268: $35-40$.

[2] Y. Ge, M. Z. E. B. Elshafie, S. Dirar, and C. R. Middleton, "The response of embedded strain sensors in concrete beams subjected to thermal loading," Construction and Building Materials, 2014, 70: 279-290.

[3] Z. Zhou, W. Liu, Y. Huang, H. Wang, H. Jianping, M. Huang, et al., "Optical fiber Bragg grating sensor assembly for 3D strain monitoring and its case study in highway pavement," Mechanical Systems and Signal Processing, 2012, 28: 36-49.

[4] R. Raju and B. G. Prusty, "Failure monitoring in composite structures using embedded FBG strain sensors," in International Conference on Fibre Optics and Photonics, Chennai, pp. 1-3, 2012.

[5] J. Du and Z. He, "FBG sensor for strain measurement with enhanced sensitivity by using degenerated FWM in highly nonlinear fiber," Electronics Letters, 2013, 49(22): 1399-1401. 
[6] C. Rodrigues, F. Cavadas, C. Felix, and J. Figueiras, "FBG based strain monitoring in the rehabilitation of a centenary metallic bridge," Engineering Structures, 2014, 44: 281-290.

[7] S. Li and D. Jiang, "Structural large strain monitoring based on FBG sensor," in Symposium on Photonics and Optoelectronics, Wuhan, pp. 1-4, 2009.

[8] L. Yuan, Q. Li, Y. Liang, J. Yang, and Z. Liu, "Fiber optic 2-D sensor for measuring the strain inside the concrete specimen," Sensors and Actuators A: Physical, 2001, 94(1-2): 25-31.

[9] B. Lee, "Review of the present status of optical fiber sensors," Optical Fiber Technology, 2003, 9(2): 57-79.

[10] Z. Zhou, J. Li, and J. Ou, "Interface transferring mechanism and error modification of embedded FBG strain sensors," Frontiers of Electrical and Electronic Engineering in China, 2007, 2(1): 92-98.
[11] H. Yuan, J. Yuan, and J. Du, "The sensing principle of FBG and its experimental application in structure strengthening detection," Journal of Wuhan University of Technology (Material Science Edition), 2003, 18 (3): 94-96.

[12] M. A. H. A. Ssomad, R. M. Hudzari, M. N. A. Noorrdin, S. M. Sapuan, N. Norhayati, and A. J. Soran, "Finite elemnt analysis for stress distribution of hand tool harvester," Procedia Engineering, 2013, 68: 219-224.

[13] B. Bahrami, S. Shahrbaf, B. Mirzakouchaki, F. Ghalichi, M. Ashtiani, and N. Martin, "Effect of surface treatment on stress distribution in immediately loaded dental implants - a 3D finite element analysis," Dental Materials, 2014, 30(14): 89-97.

[14] Micron Optics, Inc. SM125 optical sensing interrogator instruction manual. Atlanta, USA, 2007. 